\title{
Tracing a disk wind in NGC 3516
}

\author{
T. J. Turner ${ }^{1,2}$, J. N. Reeves ${ }^{3}$, S. B. Kraemer ${ }^{4}$, and L. Miller ${ }^{5}$
}

\author{
1 Dept. of Physics, University of Maryland Baltimore County, 1000 Hilltop Circle, Baltimore, MD 21250, USA \\ e-mail: turner@milkyway.gsfc.nasa.gov \\ 2 Code 662, Exploration of the Universe Division, NASA/GSFC, Greenbelt, MD 20771, USA \\ 3 Astrophysics Group, School of Physical and Geographical Sciences, Keele University, Keele, Staffordshire ST5 5BG, UK \\ ${ }^{4}$ Department of Physics, Catholic University of America, 620 Michigan Avenue NE, Washington, DC 20064, USA \\ 5 Dept. of Physics, University of Oxford, Denys Wilkinson Building, Keble Road, Oxford OX1 3RH, UK
}

Received 7 October 2007 / Accepted 26 February 2008

\section{ABSTRACT}

\begin{abstract}
Context. X-ray spectra of AGN often contain signatures indicative of absorption in multiple layers of gas whose ionization-state and covering fraction may vary with time. It has been unclear to date how much of the observed X-ray spectral and timing behavior in AGN can be attributed to variations in absorption, versus variations in the strengths of emission or reflection components. Diagnostics of the inner regions of AGN cannot be reliably performed until the origin of observed effects is understood.

Aims. We investigate the role of the X-ray absorbers in the Seyfert 1 galaxy NGC 3516.

Methods. Time-averaged and flux-selected spectroscopy is used to examine the behavior of NGC 3516 observed in Chandra HETG and XMM data from Oct. 2006.

Results. New H-like and He-like emission and absorption features discovered in the Fe K regime reveal a previously unknown zone of circumnuclear gas in NGC 3516 with $\log \xi \sim 4.3$ and column density $\sim 10^{23} \mathrm{~cm}^{-2}$. A lower-ionization layer with $\log \xi \sim 2$ and of similar column density is confirmed from previous observations, this layer has a covering fraction around 50\%, and changes in covering provide a simple explanation of a deep dip in the light curve that we interpret as an eclipse of the continuum due to passage of a cloud across the sight line within half a day. These inner zones of absorbing gas are detected to have outflow velocities in the range $1000-2000 \mathrm{~km} \mathrm{~s}^{-1}$, this, and constraints on radial location are consistent with an origin as part of a disk wind in NGC 3516.
\end{abstract}

Key words. X-rays: galaxies - black hole physics - galaxies: Seyfert

\section{Introduction}

The process of accretion onto a supermassive black hole feeds the so-called "active" subset of galaxies. The centers of galaxies are regions where material must exist in the strong gravity regime and in active galaxies there is also abundant radiation emerging in the X-ray bandpass from that region. The interaction of nuclear X-rays with circumnuclear gas allows us a direct probe of the extreme conditions close to an event horizon. It is widely thought that the large amount of angular momentum that accreting material must have means this material forms an "accretion disk" around the black hole, extending to within a few gravitational radii (the innermost stable orbit depending on the angular momentum of the black hole). Other significant components likely exist in the nuclear environs including a corona of high energy particles sandwiching the disk, a wind of material ablated from the disk surface and clouds of gas at various radial locations. Crenshaw et al. (2003) review the details of X-ray reprocessors along with estimates of the locations and conditions in various distinct zones.

Intrinsically narrow emission lines arising from the surface of the accretion disk or in any of the gas zones very close to the black hole will be distorted by a combination of Doppler and relativistic effects whose details can yield conditions and kinematics of the gas (see Fabian et al. 2000, for a review of effects). $\mathrm{X}$-ray spectroscopy provides a good probe of gas very close to the black hole as Fe $\mathrm{K} \alpha$ is a strong emission line, emitted in the X-ray band via fluorescence or recombination processes between 6.4-7 keV (depending on the ionization-state of the gas); this is a strong line owing to the high abundance and fluorescence yield of Fe. The high ionization potentials of the highest ionization states of iron mean that this line can be produced in regions where the gas is extremely ionized.

Fe $\mathrm{K} \alpha$ emission is commonly observed in AGN and much has been made of the diagnostic potential of the line, with many authors deriving accretion disk parameters from the line profile with important implications (Tanaka et al. 1995; Nandra et al. 1997). However, spectroscopy at the modest energy resolution currently available in the X-ray band has been plagued with ambiguity; complex absorption from ionized gas can produce curvature in the $2-6 \mathrm{keV}$ band that looks similar to that of an $\mathrm{Fe}$ emission line produced close to the event horizon of the accretion disk. Recent spectra from XMM have shown absorption lines from highly ionized ions in several AGN, allowing construction of detailed absorption models in some cases and revealing hitherto unknown zones of absorbing gas (e.g. Reeves et al. 2004). In the broad-line Seyfert 1 galaxy (BLSy1) NGC 3516 $(z=0.008836$; Keele 1996), absorption lines from a broad range of ions showed three distinct zones of gas covering the active nucleus in that case and producing much of the spectral curvature and variability in the X-ray band (Turner et al. 2005). The classic $U$ version of ionization parameter was utilized by Turner et al. (2005) Here we use the $\xi$ form of ionization parameter where $\xi=L / n r^{2}$. $L$ is the $1-1000$ Rydberg luminosity, $n$ the gas density and $r$ the absorber-source distance. We quote $\xi$ in units of erg $\mathrm{cm} \mathrm{s}^{-1}$ throughout and XSTAR tables are based on runs with micro-turbulence $\sigma=200 \mathrm{~km} \mathrm{~s}^{-1}$ unless otherwise noted. 
Ionization states found for the the gas zones present during 2001 observations can be converted into $\xi$ values assuming an illuminating intrinsic X-ray continuum $\Gamma \sim 2$ with $\alpha_{\mathrm{o}-\mathrm{x}} \sim 1.5$. Thus the 2001 results correspond to:

- a cool "UV absorber" with $\log \xi_{1} \sim-0.5, N_{\mathrm{H}} \sim 6 \times$ $10^{21} \mathrm{~cm}^{-2}$;

- an outflowing zone with $\log \xi_{2} \sim 3, N_{\mathrm{H}} \sim 10^{22} \mathrm{~cm}^{-2}$ and outflow velocity $\sim 1100 \mathrm{~km} \mathrm{~s}^{-1}$;

- a "heavy" absorber with $\log \xi_{3} \sim 2.5, N_{\mathrm{H}} \sim 10^{23} \mathrm{~cm}^{-2}$ covering $\sim 50 \%$ of the continuum.

Turner et al. (2005) found the spectral variability observed within 2001 to be consistent with the absorbing gas layers responding in ionization-state as the continuum flux varied.

Markowitz et al. (2007) presented a 2005 Suzaku observation where two zones of gas were detected: the primary absorber is a layer of low-ionization $\log \xi \sim 0.3$ and $N_{\mathrm{H}} \sim 6 \times$ $10^{22} \mathrm{~cm}^{-2}$ covering $\sim 96-100 \%$ of the source, while evidence is also found for a zone having $\log \xi \sim 3$ and $N_{\mathrm{H}} \sim 4 \times 10^{22} \mathrm{~cm}^{-2}$. It is unclear which of these zones correspond to particular layers isolated in 2001 data. Suzaku data were consistent with the presence of the UV-absorber detected during 2001, but if we want to associate the primary absorber detected by Suzaku with the outflowing layer detected in 2001, then the gas has undergone a major change in ionization-state between the two epochs; if we compare the Suzaku primary absorber with the "heavy" layer detected during 2001, then that layer would have had to have changed $\xi, N_{\mathrm{H}}$ and covering fraction. Whatever the correspondence between modeled zones Suzaku data clearly show NGC 3516 to have much larger absorption by relatively lowionization gas compared to any of the 2001 data (Markowitz et al. 2007). During 2006 Suzaku found NGC 3516 to have hardband flux (above $\sim 6 \mathrm{keV}$ ) comparable to that observed during April 2001 and thus the change in absorption seems likely to be due to the passage of a relatively cool cloud into the sight-line, as seen in X-ray observations of some other AGN (e.g. NGC 4151, Kraemer et al. 2005; NGC 1365, Risaliti et al. 2007). At this point one could only conclude that the inner zones of the absorber in NGC 3516 change dramatically over time in all senses.

X-ray observations of NGC 3516 also provided the first evidence for "transient" narrow emission lines, most easily explained as Doppler shifted Fe emission (Turner et al. 2002; Iwasawa et al. 2004). With all of these new discoveries our picture of the contributions from various reprocessors is rapidly evolving.

In this paper we present data from a coordinated observation of NGC 3516 utilizing Chandra and XMM data, exploring further the innermost absorbing gas in this source.

\section{The Observations}

An XMM-Newton (hereafter XMM) observation of NGC 3516 was made covering 2006 Oct. 6-13 (OBSIDs 04012010401, 501, 601, 1001). Chandra observations (OBSIDs 7281,7282, $8450,8451,8452$ ) were interwoven with the XMM exposures, to provide complete coverage of the source during that period. Figure 1 shows the light curve from the two satellites, illustrating the coverage obtained and the source behavior across the observation.

EPIC data utilized the thin 1 filter and pn PrimeSmallWindow mode. Data were processed using SAS v7.0 using standard criteria and removing periods of high background. Instrument patterns $0-4$ were selected for the pn and here our analysis describes only the pn data, as these offer the best $\mathrm{S} / \mathrm{N}$ ratio of all

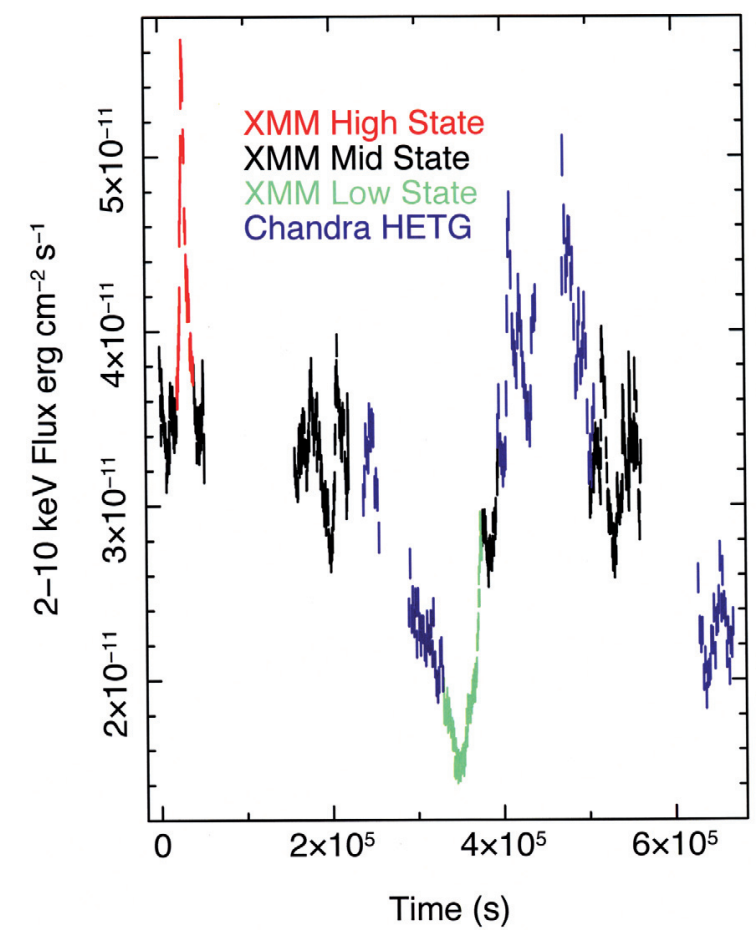

Fig. 1. XMM pn data in $500 \mathrm{~s}$ bins, and summed HEG and MEG data from all orders over $1-6 \mathrm{keV}$, in $1500 \mathrm{~s}$ bins. The light curve shows the coverage of NGC 3516 with each satellite. The XMM data were timesplit for spectral analysis, and the red points denote the time selection defining the high-flux state; the black points show the medium or midflux state, the green points show the dip event, comprising the low-flux state.

EPIC data, and were completely free of photon pileup effects. Data were extracted from a circular cell of radius $40^{\prime \prime}$ centered on the source, and background data were taken from a sourcefree region of the same pn chip. Periods where the full-band pn count rate exceeded 2 count $\mathrm{s}^{-1}$ in the background region were excluded from the analysis. The combined screening yielded a total effective EPIC pn exposure of $155 \mathrm{ks}$ over an observational baseline of about one week.

NGC 3516 gave a mean pn count rate $\sim 4.91 \pm$ 0.007 count s$^{-1}$ in the $2-10 \mathrm{keV}$ band. The mean background level in the screened data was $\sim 1 \%$ of the mean source rate in this band. As evident in Fig. 1, the source ranges across a 2-10 keV flux $F \sim 1.4-5.4 \times 10^{-11} \mathrm{erg} \mathrm{cm}^{-2} \mathrm{~s}^{-1}$ during the course of the observations. First-order RGS data yielded $0.450 \pm$ 0.001 and $0.389 \pm 0.001$ count $^{-1}$ in RGS 1 and 2, respectively. The RGS background level was $\sim 12 \%$ of the total count rate.

The Chandra HETG exposure yielded a $2-7 \mathrm{keV}$ count rate $\sim 0.021 \pm 0.0005$ count s$^{-1}$ in the summed HEG first order spectrum. Data were reduced using CIAOv3.4 and CALDB v3.4.0 and following standard procedures for extraction of HETG spectra, with the exception that we utilized a narrower extraction strip than the TGEXTRACT default. The default processing criterion cuts off the HEG data above $8 \mathrm{keV}$, yet some of the brighter and/or well-exposed sources still have good HEG data above $8 \mathrm{keV}$, including this well exposed observation of NGC 3516. This reason for the default processing cut-off is that the overlap of the MEG and HEG strips depends on the extraction strip widths, and if the latter are too large, a larger intersection of the MEG and HEG strips results, cutting off the HEG data prematurely. Specifically, we used width_factor_hetg $=20$ in the tool tg_create_mask, instead of the default value of 35 . As 


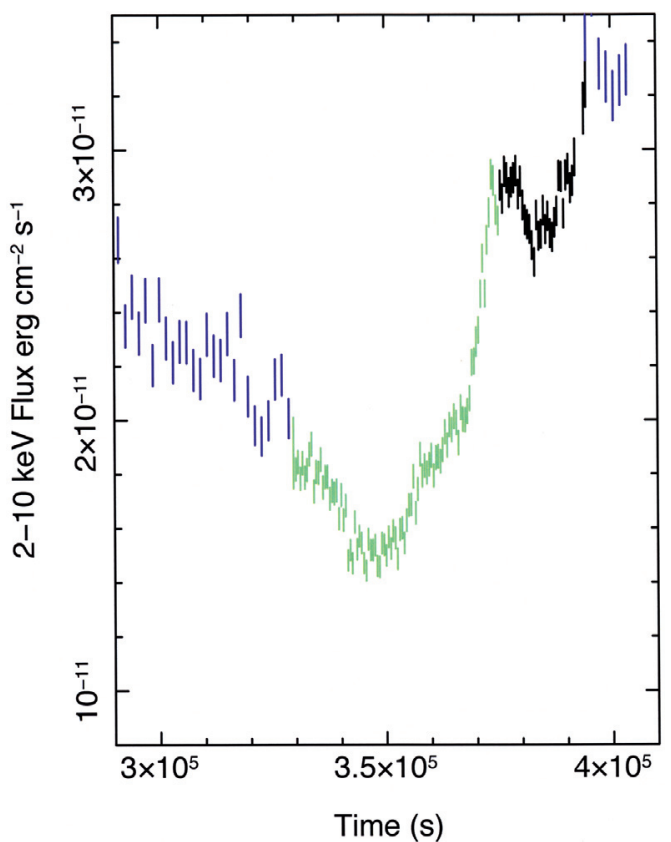

Fig. 2. A close-up view of the dip event from Fig. 1.

the HETG spectra have a very low signal-to-noise ratio it was necessary to coadd the positive and negative first-order spectra, and coadd all five OBSIDs, to create a high quality summed first-order HEG and MEG spectra for fitting. After such coaddition, we binned the spectra to 4096 channels, which yielded $>20$ counts per bin in the fitted range.

\section{Results}

The light curve from the combined observation shows the source to exhibit significant flux variability (Fig. 1), with a notable flare early in the observation, and a very sharp drop into a "dip" state later in the observation (Fig. 2). Comparison of spectra from the different states show a flattening as the source flux drops (Fig. 3) as often observed in sources of this class. Comparison of the new spectra with 2001 XMM pn and 2005 Suzaku XIS data illustrates the relatively high flux level at which the source was observed during 2006 (Fig. 4).

Inspection of the detailed profile of the light curve during the dip reveals interesting structure (Fig. 2), with the source flux levelling off for a period lasting $\sim 10 \mathrm{ks}$. Intriguingly the initial shallow dip egress has a duration of $20 \mathrm{ks}$, but breaks to a sharp rise occuring over just $6 \mathrm{ks}$.

We first analyzed the time-averaged pn spectrum. Figure 5 shows a fit to the pn data in the Fe $K$-band with 2006 data shown in red. The most obvious result is the presence of two deep absorption lines in the 6.5-7 kev regime, naturally identified with He-like and H-like species of Fe. Fitting the line pair with simple Gaussian profiles we find an equivalent width $100_{-12}^{+19} \mathrm{eV}$ for the line at $E=6.989 \mathrm{keV}$, and $45_{-7}^{+10} \mathrm{eV}$ for the line at $E=6.697 \mathrm{keV}$, both measured against the total "local continuum". To obtain the most accurate line energies and widths we turned to the contemporaneous HETG data.

Figure 6 shows the HEG spectrum of NGC 3516 from 2006. The deep absorption lines from Fe are confirmed, as are narrow emission lines in the same band. Of great interest is the detection of an emission line redward of the H-like Fe absorption feature.

Fitting the emission and absorption line "pair" close to $7 \mathrm{keV}$ yields energies close to those of rest-frame $\mathrm{H}$-like $\mathrm{Fe}$

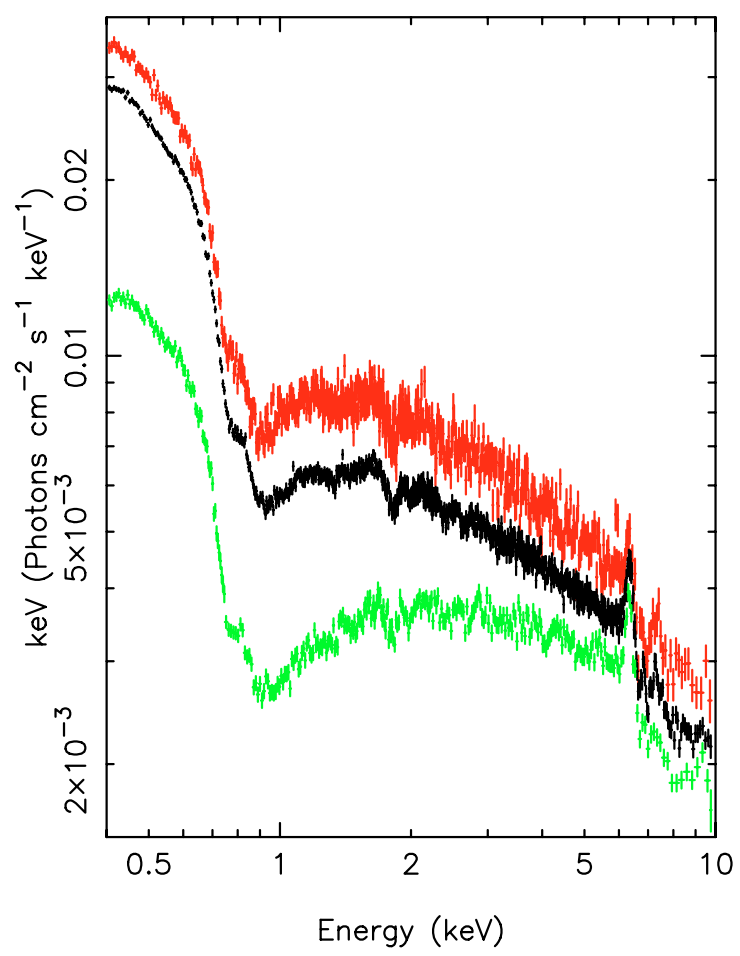

Fig. 3. X-ray spectra of NGC 3516 from XMM 2006 corresponding to the flux states denoted in Fig. 1, i.e. spectra are from the high/flare state (top), the mid-flux state (middle) and dip state (bottom).

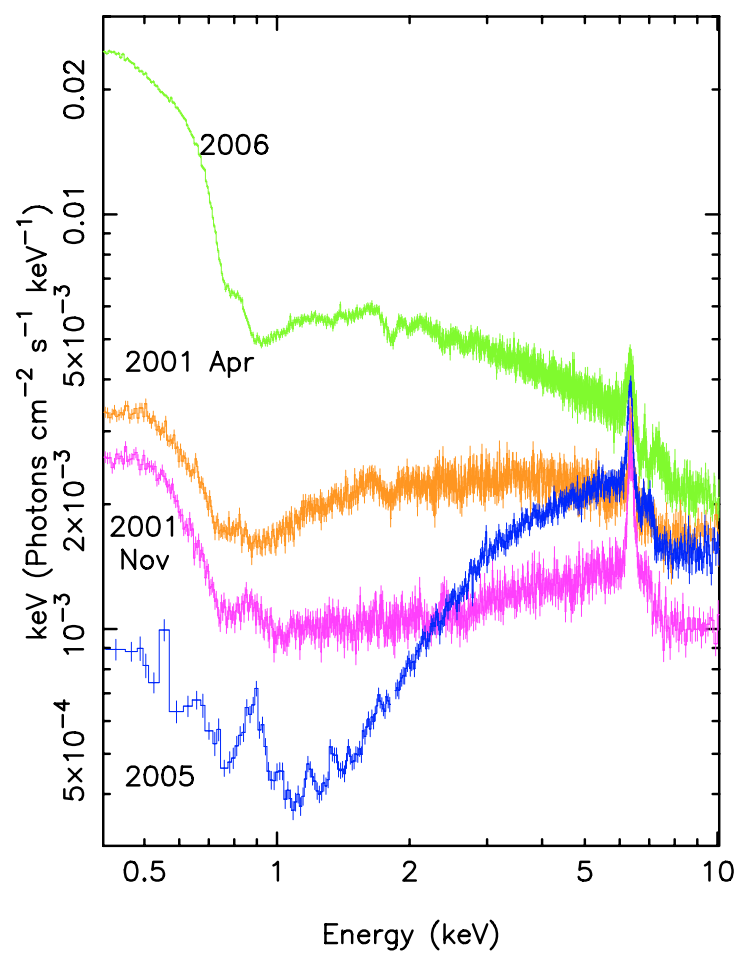

Fig. 4. X-ray spectra of NGC 3516 from XMM 2001 April, 2001 Nov., Suzaku data from 2005 October and 2006 Oct. XMM data.

transitions; the red-shifted emission and blue-shifted absorption of these components constitutes a profile somewhat akin to a classic "P Cygni" profile, indicative of an origin in an outflowing layer of gas, although in this case the emission line is offset from the expected rest energy, as well as the absorption line. A redshifted emission component might be expected if the 


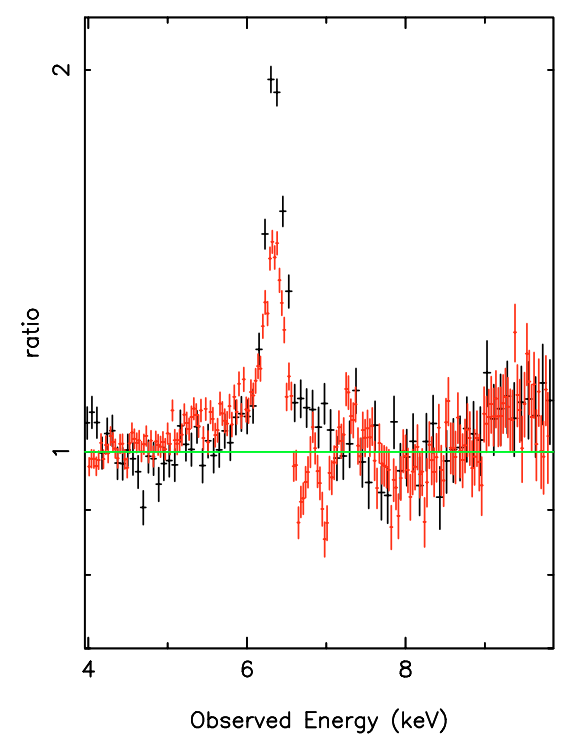

Fig. 5. $X M M$ pn data shown as a ratio to the local continuum fit in the Fe K regime during Oct. 2006 (red) and Nov. 2001 (black).

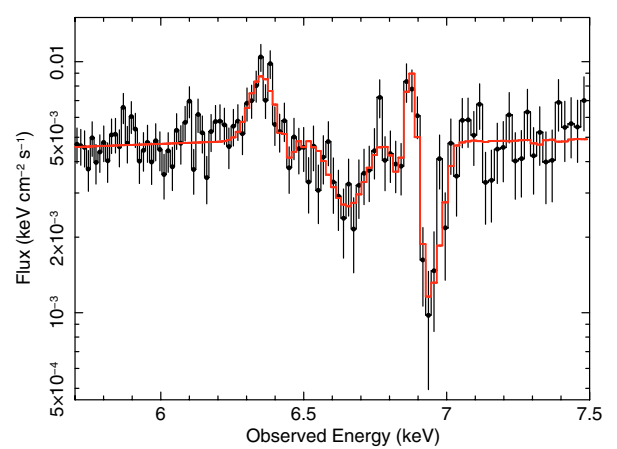

Fig. 6. Spectral data from the summed HEG first-order data from the five data segments taken during Oct. 2006. The solid line shows the model fit to the data.

outflow arises in gas that is not a simple expanding shell. A conical outflow, for example, could produce a redshifted emission component and a blue-shifted absorption component, if viewed at orientations along the flow axis of the cone. The Fe line components from $\mathrm{H}$-like $\mathrm{Fe}$ are consistent with an origin in an outflow of velocity of $\sim 1000 \mathrm{~km} \mathrm{~s}^{-1}$. The emission component of this $\mathrm{H}$-like Fe pair is too narrow and weak to show up in the $X M M$ pn spectra, but the pn spectra taken at approximately the same time and flux-state (the mid-state, Fig. 1) are consistent with the presence of such a component.

Having tight constraints on line energies and widths from HEG we tested the 2001 XMM pn and HETG data for consistency with the presence of $\mathrm{H}$-like and He-like absorption lines at the same observed energies and widths as those measured during 2006. The pn spectra were inconsistent at the $1 \%$ significance level with the presence of absorption lines at the same energies, widths and depths as observed in 2006. Specifically, the $X M M$ data limit the presence of H-like Fe absorption to be an order of magnitude weaker during Nov. 2001 and more than a factor 2 weaker during April 2001 than was observed during 2006. Figure 5 shows 2001 November pn spectral data overlaid with that from 2006, illustrating the difference in spectral signatures for the two epochs. We also analyzed the Suzaku data for NGC 3516. The data were reduced following the analysis procedure of Markowitz et al. 2007 and the XIS 0, 2 and 3 spectra

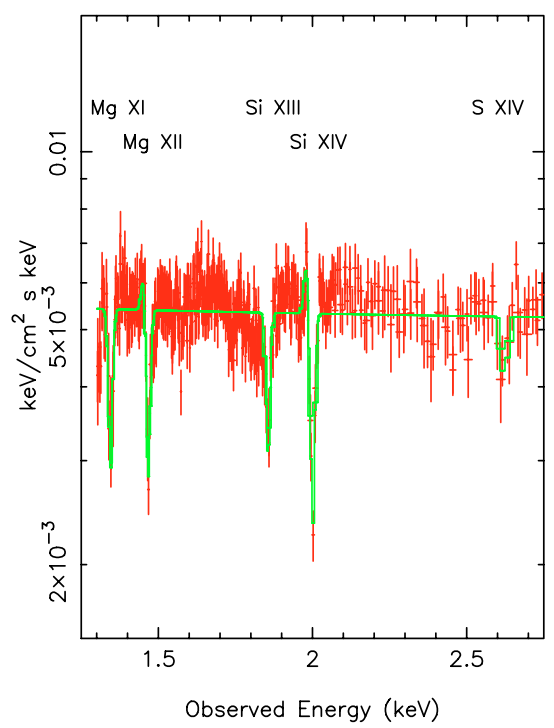

Fig. 7. Summed first-order MEG data and summed first-order HEG data from the five segments accumulated during Oct. 2006. The two grating datasets are shown coadded, the solid line shows the model detailed in Table 1 .

were summed for spectral fitting. The data were fit for the presence of the $\mathrm{H}$-like $\mathrm{Fe}$ absorption line and fitting ruled out the presence of a line of the same flux or equivalent width as observed during 2006, at the $1 \%$ significance level (assuming the line energy and width to be as detected by HETG).

We then searched the low energy part of the 2006 HETG data, fitting MEG and HEG data together to search for narrow features. Several lines are significantly detected (Fig. 7) and were modelled with a simple Gaussian form. Table 1 lists lines detected across $\sim 1-8 \mathrm{keV}$ in HETG data. Line energies tabulated have been corrected for the systemic redshift $(z=$ 0.008836). Photon fluxes are given with positive values indicating an emission line and negative an absorption line; equivalent widths are measured relative to the local observed continuum. The velocity implied for the gas is noted for each detected line, where negative velocity indicates outflow (no velocity is given for the $6.7 \mathrm{keV}$ line as this is an uncertain blend of ionization states). The last column of Table 1 shows the $\chi^{2}$ value when the line is added to the model. Quoted errors are $90 \%$ confidence intervals. The $\mathrm{Fe} \mathrm{K} \beta$ line in Table 1 was scaled to the $\mathrm{K} \alpha$ component but was not significantly detected in its own right. The $\mathrm{K} \beta$ energy was fixed appropriate to an origin in neutral gas and constrained to have flux $11.3 \%$ that of the neutral $\mathrm{Fe} \mathrm{K} \alpha$ line component. For groups of lines with closely-spaced energies we linked the line widths together in the fit so a turbulent velocity could be estimated based upon several lines together; for lines whose widths were linked the same value is shown for the $\sigma$ entry for each but only the first occurrence in Table 1 carries the combined error. Fixed parameters are denoted (f) in the table.

The fitted widths correspond to velocity dispersion $\sigma_{\mathrm{Mg}}=$ $1245_{-138}^{+160} \mathrm{~km} \mathrm{~s}^{-1}, \sigma_{\mathrm{Si}}=1265_{-176}^{+187} \mathrm{~km} \mathrm{~s}^{-1}$ and $\sigma_{\mathrm{S}}=$ $1292_{-625}^{+751} \mathrm{~km} \mathrm{~s}^{-1}$ : so, together the lines indicate a velocity dispersion $\sigma \sim 1300 \mathrm{~km} \mathrm{~s}^{-1}$. The weighted mean outflow velocity from the absorption line measurements is $\sim 1575 \pm 254 \mathrm{~km} \mathrm{~s}^{-1}$.

We examined the RGS data in the $1-2 \mathrm{keV}$ band to seek confirmation of the features detected. RGS data confirm the presence of Mg XI and XII absorption lines (absence of each is rejected at $p<1 \%$ significance), yielding line energies $E=1.358_{-0.002}^{+0.004}, E=1.482 \pm 0.005 \mathrm{keV}$ with corresponding 
Table 1. Spectral lines detected in HETG data.

\begin{tabular}{|c|c|c|c|c|c|c|}
\hline $\begin{array}{l}\text { Energy } \\
(\mathrm{keV})\end{array}$ & $\begin{array}{l}\sigma \\
(\mathrm{eV})\end{array}$ & $\begin{array}{c}n \\
\left(10^{-5} \text { photon }\right. \\
\left.\mathrm{cm}^{-2} \mathrm{~s}^{-1}\right)\end{array}$ & $(\mathrm{eV})$ & ID & $\begin{array}{c}v \\
\left(\mathrm{~km} \mathrm{~s}^{-1}\right)\end{array}$ & $\Delta \chi^{2}$ \\
\hline $1.3567 \pm 0.002$ & $6.14_{-0.66}^{+0.79}$ & $-3.17_{-0.77}^{+0.76}$ & -8 & Mg XI & $-991 \pm 440$ & 57 \\
\hline $1.4658 \pm 0.007$ & 6.14 & $0.75_{-0.50}^{+0.52}$ & 2 & Mg XII & $1302 \pm 1440$ & 13 \\
\hline $1.4806 \pm 0.001$ & 6.14 & $-2.87_{-0.55}^{+0.73}$ & -8 & Mg XII & $-1710 \pm 201$ & 160 \\
\hline $1.8709 \pm 0.003$ & $8.51_{-1.18}^{+1.26}$ & $-2.52_{-0.56}^{+0.51}$ & -9 & Si XIII & $-911 \pm 625$ & 65 \\
\hline $1.999 \pm 0.007$ & 8.51 & $0.89_{-0.57}^{+0.63}$ & 3 & Si XIV & $968 \pm 1050$ & 17 \\
\hline $2.018 \pm 0.002$ & 8.51 & $-3.24_{-0.66}^{+0.53}$ & -12 & Si XIV & $-2001 \pm 431$ & 301 \\
\hline $2.640 \pm 0.007$ & $11.4_{-5.5}^{+6.6}$ & $-1.46_{-1.14}^{+0.79}$ & -7 & S XIV & $-2591 \pm 719$ & 10 \\
\hline $6.404 \pm 0.019$ & $40.0_{-15}^{+10}$ & $5.55_{-1.76}^{+1.97}$ & 94 & $\mathrm{Fe} I$ & $-200 \pm 690$ & 31 \\
\hline $6.700 \pm 0.06$ & $62.0_{-93}^{+35}$ & $-3.74_{-1.81}^{+1.89}$ & -60 & Fe Xx & - & 14 \\
\hline $6.940 \pm 0.02$ & $16.0_{-16}^{+8}$ & $3.61_{-1.88}^{+5.73}$ & 89 & Fe XXVI & $1297 \pm 827$ & 6 \\
\hline $6.990 \pm 0.04$ & $54.0_{-10}^{+15}$ & $-6.13_{-4.24}^{+1.50}$ & -112 & Fe XXVI & $-860 \pm 1654$ & 22 \\
\hline 7.050 (f) & 40.0 & f & 14 & $\mathrm{Fe} \mathrm{I} \mathrm{K} \beta$ & & 1 \\
\hline
\end{tabular}

Table 2. X-ray absorber parameters.

\begin{tabular}{|c|c|c|}
\hline Zone & $\begin{array}{l}\text { Column density } \\
\left(10^{22} \text { atoms } \mathrm{cm}^{-2}\right)\end{array}$ & $\log \left(\xi / \operatorname{erg~cm~s}^{-1}\right)$ \\
\hline 1 & $0.24_{-0.02}^{+0.03}$ & $-2.43_{-0.03}^{+0.58}$ \\
\hline 2 & $0.05_{-0.01}^{+0.01}$ & $0.25(\mathrm{f})$ \\
\hline 3 & $20.2_{-3.2}^{+8.7}$ & $2.19_{-0.07}^{+0.07}$ \\
\hline 4 & $26.2_{-8.7}^{+6.3}$ & $4.31_{-0.14}^{+1.19}$ \\
\hline
\end{tabular}

bulk velocities 1239 and $2000 \mathrm{~km} \mathrm{~s}^{-1}$ for the outflows. The data are consistent with the detection of the Si XIII absorption line but RGS has so little area at this energy that no useful line constraints could be obtained. RGS has a slightly lower energy resolution than the HETG and could not detect the weak emission lines in this regime.

To put the new results into context, we now fit the broadband pn spectrum utilizing models for the photoionized gas run from XSTAR v21ln (Kallman et al. 2004). As this source is known to show a large degree of flux-related spectral variability we separated the pn data into high, mid and low-state spectra, as indicated in Fig. 1. For the initial fit we took the spectral data encompassed by the mid-flux state and fit across $1-10 \mathrm{keV}$. Data in this band require inclusion of four layers of gas in addition to a small column of neutral gas fixed at the Galactic line-ofsight value $\left(N_{\mathrm{H}}=4.08 \times 10^{20} \mathrm{~cm}^{-2}\right.$, Dickey \& Lockman 1990). Table 2 details the results of fitting the pn spectra covering the mid-level flux (note that the constraints on the ionization parameter of zone 4 were unique in being derived from the HEG and not the pn fit).

The two low- $\xi$ layers of gas show signatures in the RGS data below $1 \mathrm{keV}$ and provide some of the broad spectral curvature. These two layers (Table 2: zones 1 and 2) show a similar total column density to that typically detected in the $U V$ band for NGC 3516. No significant features are expected from zones 1 or 2 above $\sim 1 \mathrm{keV}$ and as this gas is likely to exist relatively distant from the active nucleus we do not discuss those zones in detail in this paper.
In the pn fit we found all layers of gas to be consistent with full covering of the source except for zone 3 (Table 2) whose covering fraction is $45 \pm 4 \%$ for gas with $N_{\mathrm{H}} \sim 2 \times$ $10^{23} \mathrm{~cm}^{-2}, \log \xi \sim 2$. The most extreme conditions detected are in a layer producing the He-like and H-like Fe absorption lines where the line depths can be fit with a zone of column density $N_{\mathrm{H}}=2.62 \times 10^{23} \mathrm{~cm}^{-2}$. Overall this 4-zone model gave $\chi^{2}=2093 / 1746$ d.o.f. with most of the contribution to $\chi^{2}$ remaining in the soft-band near $2 \mathrm{keV}$. This may be indicative of a need to refine the model but as additional features indicated by the pn close to $2 \mathrm{keV}$ do not show up in the contemporaneous HETG data. and as this is also a region most prone to pn calibration uncertainties we do not attempt to further define the model based on that part of the spectrum.

Having found a reasonable solution for the source it is interesting to revisit the individual lines detected in HETG. Examination of the XSTAR solution for zones 3 and 4 is particularly interesting. Zone 4 is of such high ionization-state that little absorption arises from that gas below the Fe $\mathrm{K}$ regime. It is most likely that the lines detected in the $1-3 \mathrm{keV}$ band arise in zone 3 , the partially-covering absorber. We conclude from the combined HETG and pn results that the partially-covering zone 3 gas has an intermediate ionization-state and large column composed of clouds with velocity widths $\sigma \sim 1210 \pm 1210 \mathrm{~km} \mathrm{~s}^{-1}$ and bulk outflow velocity $\sim 1575 \pm 254 \mathrm{~km} \mathrm{~s}^{-1}$.

A model utilizing an ionized reflector (parameterized using the REFLION model of Ross \& Fabian 2000) in place of zone 3 , but allowing in the fit the other three layers of warm absorbers did not fit the mid-state spectrum at all well $\left(\chi^{2}=\right.$ $3441 / 1714$ d.o.f.). While some reflection from the absorbing clouds must (at least) be present in this source it appears the absorption effects are dominating the spectral shape and any additional reflection was not discernible in these data. XMM lacks the broad bandpass needed to separate out any reflection component from the absorbers and reflection-dominated models are not considered further in this paper.

\subsection{Spectral variability}

Having confirmed the previously-established picture for NGC 3516 (Turner et al. 2005) as a source dominated by layers 
of complex absorbing gas, we then investigated the spectral variability of the source in the context of such a model.

First, we attempted to model the spectral steepening with flux by allowing each of the key absorber parameters (ionizationstate, column density and covering fraction) for each layer to vary in turn. We found only one way in which a variation of a single fit parameter could adequately model spectra exhibited over the full flux range traversed by the source and that was a change in covering fraction of zone 3 . As noted previously, the mid-state data represent a covering fraction of $45 \pm 4 \%$ for zone 3. A good fit can be achieved for the high-state spectrum by allowing a simple drop in covering fraction to $37 \pm 5 \%$ and for the low state by requiring an increase to $67 \pm 7 \%$ for zone 3 . All other single-parameter explanations for the observed spectral variability could be ruled out at $p<0.1 \%$ significance (mostly failing to fit the low state). Specifically, the popular model where the relative strengths of a variable power-law and steady reflection component change cannot account for the deep dip spectrum (this is ruled out at $p<0.1 \%$ significance). In the context of the variable-covering model for spectral variability then the $S u z a k u$ observation (Markowitz et al. 2007) caught NGC 3516 hidden by a large cool cloud - the overall behavior of NGC 3516 appears to be a mixture of cloud passages with changes in ionization-state of the absorbing zones. What is perhaps most surprising is the rapidity of some of the changes observed.

Using crude time-resolved spectroscopy we found that for both the flare and dip events, the change in covering fraction accounts for all but $15 \%$ of the source flux variations that are observed, leaving a question as to whether all observed variability can be caused by simple absorption changes. We therefore constructed a softness ratio to investigate the spectral variability at finer timescales than allowed by time-resolved spectroscopy. Figure 8 shows the softness ratio $\frac{0.5-4.0}{4.0-10.0} \mathrm{keV}$ count rate versus the total $0.5-10.0 \mathrm{keV}$ count rate compared to a model line representing the values allowed by our multi-zone model, for different values of covering fraction (assuming all other parameter values to be fixed, including intrinsic continuum normalization). The model line terminates at the point where the source is completely uncovered. The source behaviour is broadly consistent with the model line in the range range $10-15 \mathrm{ct} / \mathrm{s}$. Below $10 \mathrm{ct} / \mathrm{s}$ there is some residual soft flux representing some unmodeled soft emission. Above $15 \mathrm{ct} / \mathrm{s}$ there are some data showing the source to be softer than expected, these data may represent times when the ionization-state of one of the gas layers has increased (the ionization-state of absorbers laying outside of zone 3 would be expected to increase as the covering fraction of zone 3 went down because of changes in the incident illuminating flux). Flux changes above $20 \mathrm{ct} / \mathrm{s}$ are consistent with no spectral variation and may represent intrinsic continuum variability.

Finally, we note the presence of weak transient Fe emission features in the spectra, of the type first discovered in the 2001 observation of this AGN (Turner et al. 2002). The addition of a narrow $(\sigma \sim 3 \mathrm{eV})$ Gaussian emission line at $E=6.20 \pm$ $0.02 \mathrm{keV}$ was required in the pn data (absence of a line being rejected at $p<1 \%$ significance), with line flux $n=9.4 \pm 1.4 \times$ $10^{-6}$ photons $\mathrm{cm}^{-2} \mathrm{~s}^{-1}, E W=23 \pm 4 \mathrm{eV}$. Addition of a second line to the model yielded $E=5.98 \pm 0.03 \mathrm{keV}, n=6.0 \pm 2.0 \times$ $10^{-6}$ photons $\mathrm{cm}^{-2} \mathrm{~s}^{-1}, E W=15 \pm 5 \mathrm{eV}$. Some residuals are also evident in the HEG spectrum at those energies supporting the reality of the features (Fig. 6). Interestingly, the features show up most prominently in the high-state spectrum (Fig. 3), as they did during the 2002 observation. This tendency for Doppler-shifted Fe emission lines to show up in source high flux states supports a

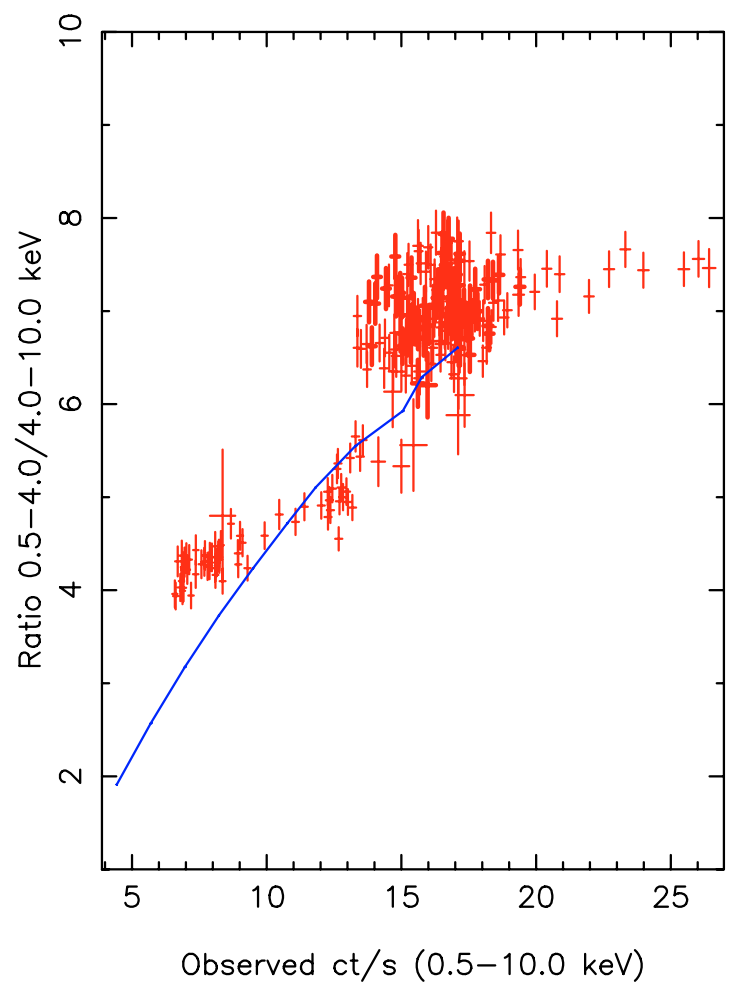

Fig. 8. The ratio $0.5-4.0 \mathrm{keV} / 4.0-10.0 \mathrm{keV}$ cts on the $y$-axis versus the total pn count rate $0.5-10.0 \mathrm{keV}$ on the $x$-axis. The solid model line shows the changes expected if the only variable is the covering fraction of zone 3 gas, the model line terminates at the point where none of the continuum source is covered by zone 3 .

close link to the continuum source flux with little lag, ie an inner disk origin.

\section{Discussion}

Here we detect four distinct layers of ionized gas absorbing the nuclear continuum. The three zones detected during 2001 have obvious counterparts in 2006 data, and a new, highly ionized layer is detected for the first time in this source. In our new analysis we also find two low-ionization zones of gas, similar to those known to cause absorption in the UV regime, and as these likely arise quite far from the nucleus, are not the topic of detailed discussion here. We concentrate on the implications of the findings for the innermost zones 3 and 4.

\subsection{The partial-covering gas: zone 3}

The "heavy" partial-covering zone detected by Turner et al. (2005) is very similar in all respects to the layer denoted "zone 3" here: this is a zone characterized by intermediate ionization-state $(\log \xi \sim 2)$, large column density $\left(N_{\mathrm{H}} \sim 2 \times\right.$ $10^{23} \mathrm{~cm}^{-2}$ ) and modest covering fraction, varying around $\sim 45 \%$, similar to that observed in 2001.

The source light curve shows a sharp, deep dip in flux having an egress of duration of $\sim 30 \mathrm{ks}$. Time resolved spectroscopy indicates that the simplest explanation of the spectral variability observed during the dip is a simple change in covering fraction of the zone 3 absorber. In the context of this model the Suzaku spectra may be interpreted as a long duration eclipse by a cool gas zone, leading to an association of the Suzaku "primary absorber" (Markowitz et al. 2007) with zone 3. Examination of the 
softness-flux plot (Fig. 8) indicates that while covering changes in zone 3 may cause deep observed dips in X-ray flux, other important phenomena are probably at work to produce the total light curve and spectral variations observed in the source. Possibilities include ionization changes of the absorber and some intrinsic variation of the source continuum flux.

The evidence for rapid changes in covering fraction of an X-ray absorber places this BLSy1 galaxy in the company of sources such as the narrow-line Seyfert 1 (NLSy1) galaxies MCG-6-30-15 (McKernan \& Yaqoob 1998), Mrk 766 (Turner et al. 2007), and the BLSy1 1H0419-577 (Pounds et al. 2004); all of which have been discussed by some authors in terms of variable absorption. Partial-covering models have been raised at various epochs as a possible explanation for Seyfert spectral and timing properties (e.g. Boller et al. 1997), the complete ambiguity between absorption and reflection dominated models in low-resolution X-ray spectra available to date left it unclear which process was most dominant. However, recent results have shown that the role of absorption has not been adequately accounted for and consideration of rapid absorption variability is obviously key to any meaningful analysis of the timing and spectral variability properties of active galactic nuclei.

The presence of large amounts of optically-thick gas close to the active nucleus means that both absorption and reflection from that material must contribute to the observed spectrum and in isolating key events, careful decomposition of the data train may lead to significant new insight into the nature of the reprocessed emission.

As noted previously, the detailed shape of the dip event in NGC 3516 is complex (Fig. 2). Some fraction of the source is completely covered for $10 \mathrm{ks}$ and then the egress begins with a $20 \mathrm{ks}$ shallow recovery followed by a rapid rise over $6 \mathrm{ks}$. The shape of the deep dip is strikingly similar to that observed for a deep dip in MCG-6-30-15 (McKernan \& Yaqoob 1998), indicating that the same physical process is likely responsible for deep flux dips across the Seyfert population.

If one considers a spherical cloud of gas of constant density occulting a point continuum source, although the cloud shape produces some gradation in opacity as the blob traverses the sight-line, this crossing would produce a smoothly varying light curve, and symmetric in ingress/egress times, rather than the asymmetric curve that is observed.

As suggested for MCG-6-30-15 by McKernan \& Yaqoob (1998) the dip profile shape can be explained by a continuum source having non-uniform emissivity. If the emitter is composed of a central bright source embedded in a fainter extended emission region, one could get a dip profile similar to that observed as a cloud crosses the line-of-sight. McKernan \& Yaqoob (1998) sketch a schematic of the general picture (their Fig. 2), applicable also to NGC 3516 (although we require no gap between the central and extended components of emission). The dip profile observed in NGC 3516 is consistent with the McKernan \& Yaqoob (1998) scenario. Another possibility is that inhomogeneities exist in the absorber, and that this complex absorber crosses a simple point-like continuum source. The asymmetry of the ingress/egress timescales gives us some additional information: these can arguably be more naturally produced with an inhomogeneous absorber crossing the sight line than with a simple cloud crossing a spatially-complex emitter. In the latter case we would need to invoke additional complexity such as that the central source is asymmetric and that the occulting material does not simply cross the source but maybe changes trajectory during its passage across the line-of-sight egressing across a different spatial dimension of the continuum emitter than it ingressed across. Complex situations such as this might arise if a cloud of gas is lifted up off the disk as it orbits, i.e. if the absorber is part of a disk wind.

HETG data trace individual lines arising in zone 3, showing a mean outflow velocity $\sim 1600 \mathrm{~km} \mathrm{~s}^{-1}$, similar to the outflow velocity $\sim 1100 \mathrm{~km} \mathrm{~s}^{-1}$ found for what appears to be the same zone observed during 2001. If this zone appears to be occulting the central emission/reflection regions then the gas must have radial and transverse components of motion.

As the transverse component of velocity is unknown, we express our derived quantities in units likely applicable to such sources. Using a mass estimate for the central black hole, $M=$ $2.95 \times 10^{7} M_{\odot}$ (Nikolajuk et al. 2006) we obtain an estimate of the radial location of zone $3, R \simeq 3.8 \times 10^{15} v_{4}^{-2} \mathrm{~cm}$ where $v_{4}$ is the orbital velocity in units of $10^{4} \mathrm{~km} \mathrm{~s}^{-1}$. For a velocity of $10^{4} \mathrm{~km} \mathrm{~s}^{-1} R \sim 1.5$ light days, locating the material on the inner edge of the BLR (Wanders et al. 1993).

The fitted ionization parameter, $\xi$ may be related to the radial location of the gas zone, $R$, the gas density $n$ and the (intrinsic) ionizing luminosity using $\xi=L_{\text {ion }} / R^{2} n$ and thus can be used to estimate $n$ (these, and other simple relationships used here are derived in detail by Crenshaw et al. 2003 and Blustin et al. 2004). The ionizing luminosity utilized by XSTAR is defined over the 1-1000 Ryd band. The observed luminosity is $L_{\mathrm{obs}} \simeq 1.2 \times$ $10^{43} \mathrm{erg} \mathrm{s}^{-1}$; correcting for the fitted absorption layers gives an implied intrinsic luminosity $L_{\text {ion }} \simeq 4.8 \times 10^{43} \mathrm{erg} \mathrm{s}^{-1}$. Utilization of the implied intrinsic luminosity gives $n \simeq 2.1 \times 10^{10} v_{4}^{4} \mathrm{~cm}^{-3}$. Combining this with the relation $N_{\mathrm{H}} \simeq n \Delta R$ where $\Delta R$ is the shell thickness, we obtain $\Delta R \simeq 9.2 \times 10^{12} v_{4}^{-4} \mathrm{~cm}$ and $\Delta R / R \simeq 2.6 \times$ $10^{-3} v_{4}^{-2}$, indicating zone 3 to be a thin shell of gas.

For zone 3 , the estimate of radial location and comparison of assumed transverse and measured outflow velocity indicates that the gas could be gravitationally bound. While a transverse velocity of order $\sim 10^{4} \mathrm{~km} \mathrm{~s}^{-1}$ is necessary to obtain a reasonable geometry, the value is subject to large uncertainty. Further, as we only measure the outflow component along our line-of-sight the true outflow velocity may be larger than observed and zone 3 is thus consistent with having comparable transverse and outflow velocities. In addition to this geometrical consideration we note that steady-state wind model solutions exist where there is continuous injection or conversion of momentum into the wind, and it is not strictly necessary to have a measured outflow velocity that is greater than the local escape velocity for an outflow ultimately to be unbound. Hence we cannot at this stage make a definite statement about whether the observed outflow forms part of a large-scale wind or whether it indicates a more complex gravitationally-bound flow.

The mass in the absorber can be estimated from $M \simeq$ $4 \pi R^{2} N_{\mathrm{H}} m_{\mathrm{p}} C_{\mathrm{g}}$ where $m_{\mathrm{p}}$ is the mass of a proton and $C_{\mathrm{g}}$ is the global covering fraction. The mass in zone 3 is estimated as $M \simeq 0.03 v_{4}^{-4} C_{\mathrm{g}} M_{\odot}$. The mass flow rate is then $\dot{M} \simeq M / t_{\mathrm{c}}$ (Crenshaw et al. 2003) where $t_{\mathrm{c}} \simeq \Delta R / v$ is the radial travel time and here $v$ is the outflow velocity rather than the orbital velocity. As noted above, for zone 3 we have measured an outflow velocity of $1600 \mathrm{~km} \mathrm{~s}^{-1}$ that is is a lower limit on the actual outflow velocity, as it is only the component of velocity that is in our line-of-sight. We estimate the mass flow rate as $\dot{M} \simeq 16 v_{1600} C_{\mathrm{g}} M_{\odot}$ year $^{-1}$, this is quantity that does not depend on the unknown orbital velocity.

Assuming the dip event was caused by a simple cloud, its diameter $d_{\text {cloud }} \sim v_{\text {orb }} t$, where $t$ is taken as the time for the egress, the strongest constraint within the dip event so $d_{\text {cloud }} \simeq 3 \times$ $10^{13} v_{4} \mathrm{~cm}$. For a velocity of $10^{4} \mathrm{~km} \mathrm{~s}^{-1}$ the lateral dimension 
of the cloud $\left(d_{\text {cloud }}\right)$ is then approximately equal to the cloud depth $\Delta R$ and a reasonable cloud geometry is thus consistent with the likely orbital velocity. The maximum coverage by the absorber is $\sim 70 \%$ suggesting the continuum to be $\sim 1.2$ times the size of cloud source on the sky. The light-crossing time for the continuum source is thus $\sim 10^{3} v_{4} \mathrm{~s}$, consistent with observed variability.

\subsection{The highly ionized gas: zone 4}

The highly-ionized zone 4 gas detected here has $\log \xi \sim 4.3$ and column density $N_{\mathrm{H}} \sim 2.6 \times 10^{23} \mathrm{~cm}^{-2}$, adding to the evidence for large columns of highly-ionized gas being important in Seyfert nuclei. This gas zone is the origin of the H-like and He-like absorption lines from the $\mathrm{K}$ shell of Fe but has little signature below $\sim 6 \mathrm{keV}$. The $2001 X M M$ data are inconsistent with the presence of He-like and H-like absorption lines of the same flux.

The very high ionization and column density, combined with apparent variability on a timescale of years and the energies at which these lines are detected disfavors an origin of this gas in the warm-hot intergalactic medium. That we have detected similarly extreme zones of gas in the longest observations of AGN (Mrk 766, Turner et al. 2007; NGC 3783, Reeves et al. 2004; MCG-6-30-15, Young et al. 2005) suggests their existence could be common in active nuclei, these features are below the limit of detectability for typical short AGN exposures conducted to date.

An intriguing aspect of this extreme zone is the apparent observation of both the emission and absorption components from $\mathrm{H}$-like $\mathrm{Fe}$ in zone 4. The pair of features appears reminiscent of classic "P Cygni" profiles that arise in outflowing shells of gas such as stellar winds. In this case it is not clear that the components arise from such a simple spherical shell of gas. Most likely, the gas has some more complex geometry (such as a bi-cone). Previous claims of "associated" emission/absorption features have been made for the lower-ionization gas zones traced by soft-band X-ray features (e.g. Kaspi et al. 2002). From the velocity separation of the emission and absorption components of $\mathrm{H}$-like $\mathrm{Fe}$ the implied outflow velocity of zone 4 is $\sim 1000 \mathrm{~km} \mathrm{~s}^{-1}$ while line widths imply a velocity dispersion $\sigma \sim 2124_{-255}^{+850} \mathrm{~km} \mathrm{~s}^{-1}$ (based on the isolated H-like absorption line).

Using the equation for ionization parameter as before we estimate a density for zone $4, n \simeq 1.6 \times 10^{8} v_{4}{ }^{4} \mathrm{~cm}^{-3}$ and $\Delta R \simeq 1.6 \times 10^{15} v_{4}^{-4} \mathrm{~cm}, \Delta R / R \simeq 0.4 v_{4}^{-2}$. The mass in zone 4 is $M \sim 0.04 v_{4}{ }^{-4} C_{\mathrm{g}} M_{\odot}$. The measured outflow velocity for zone 4 is $\sim 1000 \mathrm{~km} \mathrm{~s}^{-1}$ and the mass loss rate $\dot{M} \simeq$ $0.06 v_{1000} C_{\mathrm{g}} M_{\odot}$ year $^{-1}$.

Turning to the emission signature of zone 4 , the normalization of the H-like Fe emission line can be compared to the emission predicted by XSTAR for a full shell of gas covering the entire ionizing source. This comparison shows the observed line flux to be an order of magnitude larger than predicted. Taken at face value this leads us to an estimate of the global covering factor (covering factor seen over $4 \pi$ steradians as viewed by the continuum source) to be $C_{\mathrm{g}} \sim 11_{-6}^{+17}$ for zone 4 , which is unreasonably large, especially given the mass and loss rates estimated above.

Despite the sizable uncertainty on this covering factor it is interesting to consider the ways in which the predicted emission line strength may be brought into line with that observed. Since the Fe XXVI emission is a resonance line, its strength could be increased by photo-excitation, as suggested for the resonance lines of $\mathrm{H}$ and He-like $\mathrm{O}, \mathrm{N}$, and $\mathrm{Ne}$ in several other Seyferts (e.g. Sako et al. 2000; Armentrout et al. 2007). However, there will be little enhancement by photo-excitation once the line becomes optically thick to the continuum radiation. Although there can be greater contribution from photo-excitation if the gas is turbulent, the ionic column densities are too large in this case $\left(\sim 10^{19} \mathrm{~cm}^{-2}\right)$. For example, if the absorbing gas has a microturbulence $\sigma \sim 1500 \mathrm{~km} \mathrm{~s}^{-1}$, then the predicted line flux would be twice as large as that calculated in the XSTAR table used here. However, this is not enough of an increase to bring the predicted and observed fluxes into consistency. Even allowing for the absorption-correction to the intrinsic luminosity incident upon zone 4 doesn't produce any significant increase in the predicted line flux. The recombination line depends critically on the fractions of Fe XXVI and fully ionized Fe, and there is a limit to how much recombination line can be produced in the gas.

Nevertheless, the strength of the Fe XXVI line is very sensitive to temperature, particularly since it is primarily formed by recombination. Our models predict that when that is the dominant $\mathrm{Fe}$ ion, the gas must be in the Compton-cooled regime (Krolik et al. 1981). In this case, the gas temperature depends mostly on the spectral energy distribution of the incident radiation. A weaker X-ray flux or a stronger low energy flux could result in a significantly lower temperature, hence a stronger Fe XXVI line. For example, the presence of a reserve of low energy photons, such as may occur close to the inner edge of the accretion disk, can drive the Compton temperature down. In conclusion we note that in NGC 3516 Compton cooling appears to be important.

\section{Conclusions}

Emission and absorption features are detected across the X-ray spectrum of NGC 3516 confirming the presence of at least four distinguishable layers of absorption that shape the spectrum. Emission and absorption lines are detected from H-like Fe revealing the presence of one absorbing layer with extreme properties, $\log \xi \sim 4.3, N_{\mathrm{H}} \sim 2.6 \times 10^{23} \mathrm{~cm}^{-2}$ outflowing at $\sim 1000 \mathrm{~km} \mathrm{~s}^{-1}$.

A lower-ionization zone of gas with $\log \xi \sim 2.2, N_{\mathrm{H}} \sim 2 \times$ $10^{23} \mathrm{~cm}^{-2}$ covers just $\sim 45 \%$ of the source. Variations in covering fraction of this intermediate layer account for much of the spectral and observed flux variability in the source. In particular, a deep dip in the light curve can be explained as an eclipse by a cloud of this gas and the shape of the light curve during occultation reveals that either the cloud or the emission region must be inhomogeneous. The remarkable similarities between the deep minima of NGC 3516 and MCG-6-30-15 suggest that these events might be common in Seyfert galaxies. It appears that rapid variations in absorber parameters play a much greater role in shaping the observed X-ray variability in AGN than has been appreciated to date. These occultation events may offer the best way forward for probing the details of the innermost regions of AGN.

The detection of a significant outflow velocity for the most highly-ionized gas zone in NGC 3516 suggests that at least this component of X-ray absorption originates in a wind. The small implied radial location for the highly-ionized gas rules out the putative torus as an origin for the wind, leaving a disk wind as the most likely explanation. Ruiz et al. (2005) suggest, from optical kinematic studies, that NGC 3516 appears to be viewed at a fairly high inclination, ie close to the plane of the absorbing material and accretion disk system, similar to NGC 4151 (Crenshaw et al. 2000). Sources viewed at shallow angles to the plane of the 
disk would be the best candidates in which to view disk winds, and this may have contributed to the easy detection of outflows in highly-inclined sources such as these.

Acknowledgements. Based on observations obtained with Chandra, operated by SAO on behalf of NASA, and with XMM-Newton, an ESA science mission with instruments and contributions directly funded by ESA Member States and NASA. T.J.T. acknowledges NASA grants NNX06AH97G and G07-8092X. We thank Jianning Zeng for help with the Chandra data reduction and the anonymous referee for comments that improved the paper.

\section{References}

Armentrout, B. K., Kraemer, S. B., \& Turner, T. J. 2007, ApJ, 665, 237 Blustin, A. J., Page, M. J., Fuerst, S. V., Branduardi-Raymont, G., \& Ashton, C. E. 2005, A\&A, 431, 111

Boller, Th., Brandt, W. N., Fabian, A. C., \& Fink, H. H. 1997, MNRAS, 289, 393

Cappi, M. 2006, AN, 327, 1012

Crenshaw, D. M., Kraemer, S. B., Hutchings, J. B., et al. 2000, AJ, 120, 1731

Crenshaw, D. M., Kraemer, S. B., \& George, I. M. 2003, ARA\&A, 41, 117

Dickey, J. M., \& Lockman, F. J. 1990, ARA\&A, 28, 215

Fabian, A. C., Iwasawa, K., Reynolds, C., \& Young, A. 2000, PASP, 112, 1145

Iwasawa, K., Miniutti, G., \& Fabian, A. C. 2004, MNRAS, 355, 1073
Kallman, T. R., Palmeri, P., Bautista, M. A., Mendoza, C., \& Krolik, J. H. 2004, ApJS, 155, 675

Kaspi, S., Brandt, W. N., George, I. M., et al. 2002, ApJ, 574, 643

Kraemer, S. B., George, I. M., Crenshaw, D. M., et al. 2005, ApJ, 633, 693

Keele, W. C. 1996, AJ, 111, 696

Krolik, J. H., Mckee, C. F., \& Tarter, C. B. 1981, ApJ, 249, 422

McKernan, B., \& Yaqoob, T. 1998, ApJ, 501, 29

Markowitz, A., Reeves, J. N., Miniutti, G., et al. 2007, PASP, submitted

Nandra, K., George, I. M., Mushotzky, R. F., Turner, T. J., \& Yaqoob, T. 1997, ApJ, 477, 602

Nikolajuk, M., Czerny, B., Ziolkowski, J., \& Gierlinski, M. 2006, MNRAS, 370, 1534

Pounds, K. A., Reeves, J. N., Page, K. L., \& O’Brien, P. T. 2004, ApJ, 616, 696 Reeves, J. N., Nandra, K., George, I. M., et al. 2004, ApJ, 602, 648

Risaliti, G., Elvis, M., Fabbiano, G., et al. 2007, ApJ, 659, 111

Ruiz, J. R., Crenshaw, D. M., Kraemer, S. B., et al. 2005, AJ, 129, 73

Sako, M., Kahn, S. M., Paerels, F., \& Liedahl, D. A. 2000, ApJ, 543, L115

Tanaka, Y., Nandra, K., Fabian, A. C., et al. 1995, Nature, 375, 659

Turner, T. J., Mushotzky, R. F., Yaqoob, T., et al. 2002, ApJ, 574, L123

Turner, T. J., Kraemer, S. B., George, I. M., Reeves, J., \& Bottorff, M. C. 2005, ApJ, 618, 155

Turner, T. J., Miller, L., Reeves, J. N., \& Kraemer, S. B. 2007, ApJ, in press [arXiv: 0708.1338T]

Wanders, I., van Groningen, E., Alloin, D., et al. 1993, A\&A, 269, 39

Young, A. J., Lee, J. C., Fabian, A. C., et al. 2005, ApJ, 631, 733 